\title{
Corrigendum
}

\section{Biologies of betrayal: Judas goats and sacrificial mice on the margins of Mexico}

\author{
Emily Mannix Wanderer
}

BioSocieties (2015) 10, 116. doi:10.1057/biosoc.2015.2

Correction to: BioSocieties advance online publication 12 May 2014.

doi: 10.1057/biosoc.2014.13

A figure was introduced in this essay without sufficient attribution or permissions from the copyright holder. This has since been removed, and the remaining figures renumbered. The content and research of the article remains unchanged.

We apologise for this oversight. The corrected version of the article is published in BioSocieties 10(1). 